\title{
ESTUDO COMPARATIVO DA TOXICIDADE DO SABÃO PRODUZIDO COM ÓLEO VEGETAL RESIDUAL
}

\author{
Comparative Study of The Toxicity of Soap Made With Vegetable Oil Waste \\ Sérgio Thode Filho', Marcelo Fonseca Monteiro de Sena², Thuanny Moraes de Almeida ${ }^{3}$, \\ Vinícius da Costa Silva ${ }^{4}$ Elmo Rodrigues da Silva
}

\begin{abstract}
'Doutorando em Meio Ambiente pelo PPG-MA da Universidade do Estado do Rio de Janeiro - UERJ, Professor do Instituto Federal de Educação, Ciência e Tecnologia do Rio de Janeiro - IFRJ, Campus Duque de Caxias, RJ, Laboratório Multidisciplinar de Gerenciamento de Resíduos - LMGR

${ }^{2}$ Professor do Instituto Federal do Rio de Janeiro - IFRJ, Campus Duque de Caxias - RJ- LMGR

${ }^{3}$ Técnóloga em Processamento de Petróleo e Gás (UNESA), bolsista pesquisadora do LMGR

${ }^{4}$ Aluno do Curso Técnico em Petróleo e Gás IFRJ, colaborador do LMGR

${ }^{5}$ Professor do PPG-MA da Universidade do Estado do Rio de Janeiro - UERJ
\end{abstract}

\begin{abstract}
Resumo
Uma das formas mais seguras de descarte do óleo vegetal residual é a entrega deste em um posto de coleta, para posterior reutilização na fabricação de biodiesel ou sabão. No entanto, ainda é superficial o conhecimento científico sobre os efeitos tóxicos do óleo vegetal residual e sabão a biota. O presente trabalho objetiva comparar, em laboratório, através de um bioensaio, para o peixe Brachydanio rerio, o valor da concentração letal média ( $\mathrm{CL}_{50}$-72horas) do sabão sólido produzido no IFRJ a partir do óleo vegetal residual, o sabão pastoso produzido com óleo vegetal residual e o sabão sólido em barra, ambos comercializados no grande varejo. Os peixes foram acondicionados em béqueres contendo oito diferentes concentrações de amostras de sabão sólido de três diferentes origens. O teste de exposição consistiu no procedimento de manter os peixes em contato por 72 horas. O sabão sólido comercial que utiliza insumos virgens possui letalidade consideravelmente menor quando comparado aos saponáceos produzidos a partir do óleo vegetal residual; enquanto que a diferença de letalidade foi inexpressiva entre os grupos de saponáceos que utilizam óleo vegetal residual.
\end{abstract}

Palavras-chave: Impacto ambiental, bioensaio, sabão, óleo vegetal residual

\begin{abstract}
One of the safest ways to dispose of residual vegetable oil is delivering this on a collection point for later use in the manufacture of biodiesel or soap. However, it is still superficial the scientific knowledge about the toxic effects of these residual waste vegetable oil and soap on biota. The present study aims to compare, by bioassay with the fish Brachydanio rerio, the median lethal concentration ( $\mathrm{CL}_{50}-72 \mathrm{hours}$ ) of solid soap produced in IFRJ from residual vegetable oil, with the pasty soap produced from waste vegetable oil and the solid bar soap, both sold in large retail. The fishes were placed in beakers containing eight different concentrations of soap samples. The test consisted of exposure of fishes for 72 hours. The solid soap commercial that uses virgin inputs has significantly lower mortality rate compared to soaps produced from residual vegetable oil, whereas the difference in mortality was insignificant between groups of soaps using residual vegetable oil.
\end{abstract}

Keywords: Environmental impact, bioassay, soap, waste vegetable oil. 


\section{INTRODUÇÃO}

Uma das questões presentes nas determinações da Política Nacional de Resíduos Sólidos, diz respeito ao descarte do óleo vegetal de frituras gerado nas residências e estabelecimentos comerciais. No Brasil estima-se a produção de três bilhões de litros de óleo vegetal comestível por ano. Deste total, apenas $2,5 \%$ é reutilizado para alguma finalidade, enquanto que o restante é indevidamente descartado, pela população e indústrias, nos solos, corpos d'água, rede de esgotos, ou ainda, incinerados (ABIOVE, 2012).

Segundo a Folha do Amapá (2007), uma forma mais segura de descarte de óleo vegetal residual é a entrega deste em um posto de coleta, para posterior reutilização na fabricação de biodiesel ou sabão. Além desta, a fabricação de sabão na própria residência tem se mostrado como uma possibilidade alternativa. A fabricação de sabão utilizando óleo vegetal residual é uma forma de evitar que este alcance os corpos hídricos e o solo na forma prejudicial, mitigando assim seus impactos no meio ambiente.

No entanto, ainda é superficial o conhecimento científico sobre os efeitos tóxicos destes resíduos de óleo vegetal residual e sabão a biota. Segundo Zagatto et al. (1988), a toxicidade é definida como sendo os resultados nocivos à saúde provenientes do sistema composto por substâncias químicas e substâncias próprias do organismo, que se evidenciam sobre organismos vivos. Logo, na toxicidade o objetivo de análise é o organismo, onde é determinado o efeito causado por uma substância química ou uma mistura, levando em consideração o tempo de exposição e a concentração.

Os bioensaios têm sido aplicados em diversos países há muitos anos, na rotina de órgãos ambientais, no âmbito do licenciamento e da fiscalização de atividades potencialmente causadoras de poluição e contaminação (KNIE; LOPES, 2004).Estes testes visam principalmente avaliar a sensibilidade relativa de organismos aquáticos para um determinado agente tóxico, para determinar a qualidade de água e as concentrações seguras de agentes químicos para preservação da vida aquática, além de avaliar a fertilidade potencial das águas e a eficiência de diferentes métodos de tratamento para efluentes industriais em termos toxicológicos (METCALF; EDDY, 2003).

O presente trabalho objetiva comparar, em laboratório, através de um bioensaio, para o peixe Brachydaniorerio, o valor da concentração letal média ( $\mathrm{CL}_{50}$-72horas) do sabão sólido produzido no IFRJ a partir do óleo vegetal residual, o sabão pastoso produzido com óleo vegetal residual e o sabão sólido em barra, ambos comercializados no grande varejo.

\section{METODOLOGIA}

Para o teste de toxidez foi utilizado o peixe paulistinha Brachydanio rerio, espécie de peixe exótica frequentemente usada em testes de avaliação toxicológica de pesticidas (PATRICIO et al., 2002) e considerada como padrão internacional para a realização deste tipo de experimento (MURTY, 1988). Os peixes foram acondicionados em béqueres contendo oito diferentes concentrações de amostras de sabão sólido de três diferentes origens: (grupo 1) sabão sólido produzido a partir do óleo vegetal residual no Laboratório Multidisciplinar de Gerenciamento de Resíduos do Instituo Federal do Rio de Janeiro campus Duque de Caxias; (grupo 2) sabão pastoso produzido om óleo vegetal residual vendido em grandes redes de varejo e (grupo 3) sabão sólido comercial produzido com glicerina e insumos virgens vendido em grandes redes de varejo.

O sabão sólido produzido a partir do óleo vegetal residual utiliza a relação $1: 2: 6$, isto é uma parte de $\mathrm{NaOH}$, duas partes de água e seis partes do óleo residual é a relação considerada ótima entre os insumos. Constatou-se que na relação utilizada, não foram encontradas presença de soda livre, presença de óleo e água que não foram totalmente utilizados no processo de reação. Percebe-se que mesmo sem a utilização de essências ou aromatizantes o produto final apresenta um aspecto físico uniforme, uma textura rígida, uma coloração amarelo pálido, um odor agradável, um índice de espuma satisfatório e um $\mathrm{pH}$ aceitável. O sabão produzido com estes insumos são indicados para uso em limpeza pesada, sendo impróprios para o asseio corporal (THODE-FILHO et al., 2013). As concentrações e os grupos estão demonstrados na Tabela 1.

Cada recipiente recebeu quatro peixes com peso individual variando entre 80 e $100 \mathrm{mg}$, respeitando-se a densidade máxima de 1,0 g.L.-1, conforme descrito por Boock (2005).

A água nos béqueres foi constantemente aerada e mantida sob temperatura em torno de $20-25^{\circ} \mathrm{C}$. Os animais foram alimentados diariamente com ração específica para peixes ornamentais, sendo observado o consumo da ração de maneira a evitar que restos se deteriorassem, 
comprometendo a qualidade da água (BOOCK \& NETO, 2005). O experimento é caracterizado como estático, pois não há substituição de água e nem retirada de detritos durante o período de exposição (BOOCK et al., 2004). O teste de exposição consistiu no procedimento de manter os peixes em contato, por 72 horas. As análises foram realizadas em triplicata para cada uma das concentrações testadas. A cada teste procedeu-se um branco (testemunha) (BOOCK et al., 2004). Os animais mortos foram contados e retirados diariamente.

\section{RESULTADO E DISCUSSÃO}

Para desenvolvimento deste bioensaio, foram adotadas diferentes concentrações do sabão sólido produzido com óleo vegetal residual do IFRJ, do sabão pastoso comercial produzido com óleo vegetal residual e do sabão sólido comercial produzido sem óleo vegetal residual em sua composição. A tabela 1 apresenta as diferentes concentrações de massa de sabão dos grupos 1, 2 e 3 .

A Tabela 1 apresenta os valores de concentração letal identificados para cada grupo experimental. Observa-se que o sabão sólido comercial que utiliza insumos virgens possui letalidade consideravelmente menor quando comparado aos saponáceos produzidos a partir do óleo vegetal residual; enquanto que a diferença de letalidade foi inexpressiva entre os grupos de saponáceos que utilizam óleo vegetal residual, ou seja, a diferença encontrada entre os grupos 1 e 2 foi de $0,005 \mathrm{~g} / 1000 \mathrm{~mL}(0,005 \mathrm{ppm})$.

Em uma observação isolada, pode-se concluir que os saponáceos comerciais que utilizam insumos virgens são menos impactantes ambientais por serem menos letais a biota. Em contrapartida, em uma perspectiva mais abrangente, observando os ciclos dos resíduos, entende-se que os saponáceos produzidos a partir do óleo vegetal residual retiram (ou impedem que seja despejado) do ambiente um agente poluidor (óleo vegetal residual) o qual é igualmente, ou mais, impactante que o saponáceo.

Para o sabão sólido produzido no IFRJ, utilizou-se $400 \mathrm{~g}$ de $\mathrm{NaOH}, 800 \mathrm{ml}$ de água e 2600 $\mathrm{ml}$ de óleo ou aproximadamente $2300 \mathrm{~g}$ de óleo vegetal residual. O rendimento pós produção é de aproximadamente $4000 \mathrm{~g}$. Para calcular a massa de óleo vegetal residual existente em $0,029 \mathrm{~g}$ de sabão do grupo 1, foi utilizado o fato de serem grandezas diretamente proporcionais e aplicada à regra de três para o cálculo. Neste sentido, a massa de óleo vegetal residual constituinte em $0,029 \mathrm{~g}$ é de $0,016 \mathrm{~g}$. Representando aproximadamente 55\% da massa total de sabão.

Tabela 1. Mortalidade de Brachydaniorerio submetidos a diferentes concentrações origens de saponáceos.

\begin{tabular}{|c|c|c|c|}
\hline \multirow{2}{*}{ Concentrações } & \multicolumn{3}{|c|}{ Mortalidade } \\
\hline & Grupo $1^{\mathrm{a}}$ & Grupo $2^{b}$ & Grupo $3^{c}$ \\
\hline $0,020 \mathrm{~g} / 1000 \mathrm{~mL}$ & - & & \\
\hline $0,025 \mathrm{~g} / 1000 \mathrm{~mL}$ & $25 \%$ & _ & - \\
\hline $0,029 \mathrm{~g} / 1000 \mathrm{~mL}^{*}$ & $50 \%$ & $25 \%$ & - \\
\hline $0,034 \mathrm{~g} / 1000 \mathrm{~mL}^{*}$ & $100 \%$ & $50 \%$ & - \\
\hline $0,040 \mathrm{~g} / 1000 \mathrm{~mL}$ & $100 \%$ & $100 \%$ & - \\
\hline $0,045 \mathrm{~g} / 1000 \mathrm{~mL}$ & $100 \%$ & $100 \%$ & - \\
\hline $0,047 \mathrm{~g} / 1000 \mathrm{~mL}$ & $100 \%$ & $100 \%$ & - \\
\hline $0,050 \mathrm{~g} / 1000 \mathrm{~mL}$ & $100 \%$ & $100 \%$ & - \\
\hline $0,060 \mathrm{~g} / 1000 \mathrm{~mL}$ & $100 \%$ & $100 \%$ & - \\
\hline $0,070 \mathrm{~g} / 1000 \mathrm{~mL}$ & $100 \%$ & $100 \%$ & - \\
\hline $0,080 \mathrm{~g} / 1000 \mathrm{~mL}$ & $100 \%$ & $100 \%$ & $25 \%$ \\
\hline $0,082 \mathrm{~g} / 1000 \mathrm{~mL}^{*}$ & $100 \%$ & $100 \%$ & $50 \%$ \\
\hline $0,085 \mathrm{~g} / 1000 \mathrm{~mL}$ & $100 \%$ & $100 \%$ & $100 \%$ \\
\hline
\end{tabular}

${ }^{\text {aS }}$ abão sólido produzido a partir do óleo vegetal residual de acordo com Thode-Filho et al. (2013).

b́abão pastoso produzido com óleo vegetal residual vendido comercialmente.

'Sabão sólido comercial produzido com glicerina e insumos virgens vendido comercialmente.

*Valor da concentração letal média $\left(\mathrm{CL}_{50}-72\right.$ horas) identificados para cada grupo experimental. 
Assim, pode-se observar que apesar do sabão produzido a partir do óleo vegetal residual ser mais letal, ele estende a vida útil de outro resíduo e reduz a utilização de insumos virgens que passam a estar disponíveis para outras finalidades.

Diante de tantos desafios, vale ressaltar os esforços governamentais para disciplinar o problema dos lançamentos em efluente sem tratamento a exemplo da Resolução CONAMA n ${ }^{\circ} 430 / 11$ que institui o padrão de lançamento em corpo líquido de óleos vegetais e gorduras animais de $50 \mathrm{mg} / \mathrm{L}$ ou $0,005 \mathrm{~g} / \mathrm{L}$ sem a presença de resíduos sólidos em suspensão (BRASIL, 2011). Percebe-se que a quantidade de óleo residual existente na massa de sabão do grupo 1 experimental é menor do que o previsto no CONAMA.

Adicionalmente, o óleo vegetal residual tem ação impactante a longo-prazo, provocando efeitos crônicos. Caso seja descartado pela rede de esgoto, pode provocar o entupimento das tubulações e aumentar em até $45 \%$ os custos de tratamento. O material pode ocasionar também sérios danos ambientais ao alcançar os corpos d'água, pois o óleo forma uma camada na superfície da água que impede a entrada da luz solar, diminuindo a fotossíntese, o oxigênio dissolvido, e, consequentemente, provoca a morte da fauna local (Qi et al., 2009).

Em contrapartida, o sabão tem ação a curto-prazo, isto é, proporciona um efeito agudo, conforme observado neste estudo, pois há alguns problemas e dificuldades no processo de obtenção do sabão a partir do óleo vegetal residual, como o longo período de tempo de reação de saponificação, saponificação incompleta, relação ótima entre os insumos, entre outros. Qi et al. (2009) mostraram que o tratamento de saponificação atingiu a configuração otimizada com quatro horas de reação à $100^{\circ} \mathrm{C}$, com a proporção de solução de hidróxido de sódio e óleo vegetal residual de 1:2.

\section{CONCLUSÃO}

Este ensaio experimental possibilita identificar que o sabão produzido com óleo vegetal residual, pode ser uma medida mitigadora para redução do impacto ambiental provocado pelo descarte inadequado do óleo vegetal residual. $\mathrm{O}$ mesmo permite que matérias primas virgens envolvidas no processo de fabricação, sejam destinadas para finalidades mais nobres.

A diferença encontrada entre os grupos $1 \mathrm{e}$ 2 é de respectivamente $0,005 \mathrm{~g} / 1000 \mathrm{~mL}$. Verifica-se que para um sabão produzido artesanalmente essa diferença torna-se aceitável, em relação a um sabão industrializado, produzido em larga escala, que utiliza o óleo vegetal residual como insumo principal, porém outros insumos virgens para estabilidade da sua composição.

Com os resultados deste estudo percebe-se a necessidade de desenvolver outros bioensaios trabalhando com o sabão do grupo 1 no mesmo estado da matéria que o grupo 2 , base pastosa.

\section{AGRADECIMENTOS}

Agradecemos ao Prof. Bruno Pereira Berto (Departamento de Biologia Animal, Instituto de Biologia, Universidade Federal Rural do Rio de Janeiro - UFRRJ) pelas sugestões e orientações na metodologia do bioensaio.

\section{REFERÊNCIAS}

ABIOVE. Associação Brasileira das Indústrias de Óleos Vegetais. 2012. Disponível em: <http:// www. abiove.com.br/menu_br.htmL >. Acesso em: 18 jan. 2013.

BOOCK, M. V., ZORZENON F. J., FARIAS A. A., SILVA, E. A., ALMEIDA, S. D. B. (2004) Toxicidade aguda do inseticida Metroprene para os peixes brachydaniorerio (paulistinha) e Poeciliareticulata (website). Arquivos do Instituto Biológico, São Paulo, v.71 (suplemento), 307, 711-713.

BOOCK, M. V., MACHADO NETO, J. G. (2005) Estudos sobre a toxicidade aguda do oxicloreto de cobre para o peixe Poeciliareticulata. Boletim Instituto de Pesca, São Paulo, (31) 1, 29-35.

BRASIL. Resolução n ${ }^{\circ} 430$ de 13 de maio de 2011. Dispõe sobre as condições e padrões de lançamentos de efluentes, complementa e altera a Resolução ${ }^{\circ}$ 357, de 17 de março de 2005, do Conselho Nacional do Meio Ambiente - CONAMA.

FOLHA DO AMAPÁ. Óleo de cozinha usado contamina solo água e atmosfera,2007. Disponível em: <http://www.folhadoamapa.com.br $>$. Acesso em: 21 jul. 2012.

KNIE, J.L.W. e LOPES, E.W.B. 2004 Testes eco toxicológicos: métodos, técnicas e aplicações. Florianopolis: FATMA / GTZ. 289p. 
METCALF, L. \& EDDY, H. P. Wastewater engineering treatment in reuse. 4.ed. Mcgraw Hill: Boston, 2003.

MURTY, A. S. Toxicityofpesticidetofish. (1988) USA, CRC press, (1), 192p.

PATRICIO, F. C., RIGITANO, R. L. O, GOUVÊA, A. V., FRANCO, A. A. (2002) Toxicidade do inseticida-nematicida Aldicarbe às espécies de peixes Brachydaniorerio e Orthospinusfranciscensis. Ciência e Agrotecnologia, Lavras, (26) 2, 385-391.

QI, D.; WANG, Q.; WANG, QI.; HUANG, Q.; YIN, P. (2009) Study on Saponification Technology of Waste Edible Oil. In Bioinformatics and Biomedical Engineering,.ICBBE. 3rd International Conference on, pp. 1-4. IEEE, 2009.

THODE-FILHO, Sergio; SENA, Marcelo Fonseca Monteiro de; SILVA, Elmo Rodrigues; CABRAL, Gabrielle Borges; MARANHÃO, Fabíola da Silveira. (2013). Sistema de análise estequiométrica para produção de sabão a partir do óleo vegetal residual: uma estratégia para redução do impacto ambiental. Revista Eletrônica em Gestão, Educação e Tecnologia Ambiental, (15) 15, 3019-3025.

ZAGATTO, P. A.; BERTOLETTI, E. GOLDSTEIN, E. G. (1988) Toxicidade de efluentes industriais da bacia do rio Piracicaba. In: Revista Ambiente, (2) 1, $39-42$.

SOUZA, S. S. Mudanças cambiais e o efeito dos fatores de crescimento das receitas de exportações brasileiras de soja. Revista de Economia e Agronegócio, Viçosa, v. 5, n. 1, p. 1-24, jan./mar. 2007. 